\title{
STIRLING ENGINE PERFORMANCE PREDICTION USING SCHMIDT ANALYSIS BY CONSIDERING DIFFERENT LOSSES
}

\author{
Rakesh K. Bumataria ${ }^{1}$, Nikul K. Patel ${ }^{2}$ \\ ${ }^{1}$ P. G. Student, Mechanical Engg. Dept., The M S University of Baroda, Gujarat, India, bumatariarakesh@yahoo.co.in \\ ${ }^{2}$ Assistant Professor, Mechanical Engg. Dept., The M S University of Baroda, Gujarat, India, nikulatmsu@gmail.com
}

\begin{abstract}
A low temperature ratio Stirling engine analysis required for cost effective, less environment harmfulness and more efficient power generation compare to Rankine cycle and Brayton cycle for this temperature ratio. A new and complete model for a Stirling engine has been established. This computerized model predicts the behavior of existing engines reasonably accurately for cases where a quantitative comparison is available. In order to obtain a closed solution suitable for design optimization a simplified model for a Stirling engine has been derived considering different types of losses in this engine. This new model has sufficient accuracy for prediction of the behavior a real engine and its results are quite close to the complete model predictions.
\end{abstract}

Index Terms: Stirling Engine, Schmidt Analysis, Low temperature ratio, Engine Losses, computerized model

\section{INTRODUCTION}

Analysis of the Stirling cycle is complicated by the fact that not all elements of the working fluid pursue the same thermodynamic cycle. Since the ideal cycle analysis has been presented in closed form solutions, and then they are useful for preliminary design and first order calculations. In this section the first order analysis (Schmidt equations) are presented with considering different types of losses in Stirling engine.

\section{IDEAL STIRLING CYCLE, SCHMIDT EQUATIONS}

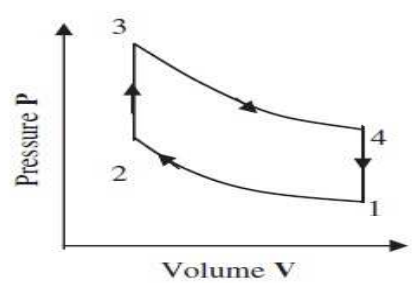

Fig-1: Ideal Stirling Cycle P-V Diagram

As shown in Fig.1, the ideal Stirling cycle consists of two isotherms connected by two isochors. These processes may be produced by interconnecting two suitably varying volumes through a regenerator. The cycle starts with isothermal compression in the cold space at cold temperature $\mathrm{T}_{\mathrm{C}}$, process 1-2. Then the gas flows through the regenerator and gains enough heat to reach the hot temperature $\mathrm{T}_{\mathrm{H}}$. This heating is such that the same volume of gas which enters to the regenerator from cold space should discharge from regenerator to hot space, i.e. the volume variations of hot and cold spaces must be appropriately related. This constraint is necessary to satisfy the isochoric heating process 2-3. Then gas expands isothermally in hot space (hot cylinder) at $\mathrm{T}_{\mathrm{H}}$, requiring heat to be added to gas to maintain it at $\mathrm{T}_{\mathrm{H}}$, process 3-4. Then the gas returns through the regenerator where heat is removed from the working fluid and stored for its subsequent return, process 4-1. So, defined engine is very idealized and impractical engine. A more realistic cycle and corresponding analysis was devised by Gustav Schmidt in 1871. This analysis which has a sinusoidal volume variation has now become the classical analysis of the cycle and is generally believed to give a more reasonable approximation of actual engine performance. Nevertheless, the analysis still remains very highly idealized, so that in practice the indicated performance of an engine will likely be no better than $60 \%$ of the predicted Schmidt cycle performance. Principal assumptions of the Schmidt cycle are [1]:

1. The regenerative process is perfect.

2. The instantaneous pressure is the same throughout the system.

3. The working fluid obeys the characteristic gas equations, PV=RT.

4. There is no leakage and the mass of working fluid remains constant.

5. The volume variations in the working space occur sinusoidal.

6. There are no temperature gradients in the heat exchangers. 
7. The cylinder wall and piston temperatures are constant.

8. There is perfect mixing of the cylinder constants.

9. The temperature of the working fluid in the dead volumes is constant.

10. The speed of the machine is constant.

11. Steady state flow conditions are established.

\section{ANALYSIS OF PRACTICAL STIRLING ENGINES}

Analysis of the Stirling engine can be divided into three groups or so called "orders" [2] first order analysis includes the basic thermodynamics of the engine on a stepwise model which is usually called the Schmidt analysis. In order to make this analysis comparable with real engine performances it should be combined with some critical experiences factors. Second order analysis starts with the Schmidt analysis or something similar. Various power losses are calculated and added to the Schmidt heat input. All these engine processes are assumed to process in parallel and independently of each other. Finally, the third order analysis divides the engine into a number of nodes and solves the basic differential equations that govern this engine by numerical methods. Third order methods are much more laborious but since fewer assumptions are made prediction of engine performance is expected to be more accurate.

In this section the method of analysis which is used for this paper is presented. This method is more accurate than the second order analysis and it is essentially based on the Rios [4] second order model. Similarity of this model and the second order method is because a calculation of the losses is independent of the basic engine thermodynamics calculations. Otherwise, it makes no use of the Schmidt model as being too idealized.

\section{MASS LOW RATES INSIDE THE STIRLING ENGINE}

One of the important tasks of modeling a Stirling engine is to present a model which is able to represent the dynamics involved in all essential processes of the engine. Determination of an accurate average mass flow rates, over a cycle inside each components of the system is important because these values determines the Reynolds's number, pressure drop and temperature drop inside the corresponding components. This constitutes one more reasons why most over-simplified closed form solutions which have been previously presented do not predict the behavior of a real Stirling engine accurately.

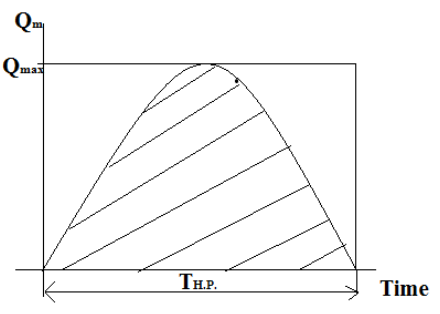

Fig-2: General Configuration of a Stirling engine

Fig. 2 shows a general configuration of a Stirling engine. Since it is assumed the total mass of the working fluid is constant and there is essentially no mass leakage, then it means whatever mass flows into the three heat exchangers in the first half of the cycle should come out of them during the second half of the cycle. The assumption of sinusoidal volume variations inside the hot and cold spaces usually helps to provide similar mass flow results.

$$
\begin{aligned}
& V_{h}=V_{H} \times \frac{(1-\sin \omega t)}{2} \\
& V_{c}=V_{C} \times \frac{[1-\sin (\omega t-\phi)]}{2} \\
& Q_{n e t}=\frac{V_{C} \times \omega \times \sin \phi}{2 \cos \alpha} \sin (\omega t+\alpha) \\
& \text { Where } \tan \alpha=\frac{\left(\frac{V_{H}}{V_{C}}+\cos \phi\right)}{\sin \phi}
\end{aligned}
$$

These pressures and temperatures would result in the following mass flow rates.

Heater:

$\dot{m}_{H}=P_{m} \times\left(1+\frac{A}{\sqrt{1-A^{2}}}\right) \times V_{C} \times \omega \times \frac{\sin \phi}{2 \times R \times T_{H} \times \cos \alpha} \times F_{c h}$

Cooler:

$\dot{m}_{C}=P_{m} \times\left(1-\frac{A}{\sqrt{1-A^{2}}}\right) \times V_{C} \times \omega \times \frac{\sin \phi}{2 \times R \times T_{C} \times \cos \alpha} \times F_{c c}$

Regenerator:

$$
\dot{m}_{R}=P_{m} \times V_{C} \times \omega \times \frac{\sin \phi}{2 \times R \times T_{R} \times \cos \alpha} \times F_{c r}
$$

Where $\mathrm{F}_{\mathrm{ch}}, \mathrm{F}_{\mathrm{cc}}$ and $\mathrm{F}_{\mathrm{cr}}$ are the correction factors, which have to be determined. As might be expected they are very close to unity. The same considerations used for basic output power correction factors can once again be applied here. This means that each of these correction factors $\mathrm{F}_{\mathrm{ch}}, \mathrm{F}_{\mathrm{cc}}, \mathrm{F}_{\mathrm{cr}}$ are functions 
of temperature ratios $\left(\frac{T_{H}}{T_{C}}\right)$, phase angle differences $\phi$ and dead volume ratios. Therefore, each of them can be taken as a multiplication of three factors.

$$
\begin{aligned}
& F_{c c}=f_{c t}(T) \times f_{c \phi}(\phi) \times f_{c D}(D) \\
& F_{c r}=f_{r t}(T) \times f_{r \phi}(\phi) \times f_{r D}(D) \\
& F_{c h}=f_{h t}(T) \times f_{h \phi}(\phi) \times f_{h D}(D)
\end{aligned}
$$

Different correction factors for mass flow rate are as follow.

For temperature ratio $\left(\frac{T_{H}}{T_{C}}\right)$ :

Heater:

$$
f_{h t}(T)= \begin{cases}-0.24\left(\frac{T_{H}}{T_{C}}\right)^{2}+0.187\left(\frac{T_{H}}{T_{C}}\right)+0.8455 & ; \frac{T_{H}}{T_{C}} \geq 1.5 \\ 0.205\left(\frac{T_{H}}{T_{C}}\right)+0.7645 & ; \frac{T_{H}}{T_{C}}<1.5\end{cases}
$$

Cooler:

$$
f_{c T}(T)= \begin{cases}0.01962\left(\frac{T_{H}}{T_{C}}\right)^{2}-0.2088\left(\frac{T_{H}}{T_{C}}\right)+1.3102 & ; \frac{T_{H}}{T_{C}} \geq 1.5 \\ -0.178\left(\frac{T_{H}}{T_{C}}\right)+1.309 & ; \frac{T_{H}}{T_{C}}<1.5\end{cases}
$$

Regenerator:

$$
f_{r T}(T)= \begin{cases}1.052 & ; \frac{T_{H}}{T_{C}}>2 \\ -0.0194\left(\frac{T_{H}}{T_{C}}\right)^{2}+0.0923\left(\frac{T_{H}}{T_{C}}\right)+0.943 & ; \frac{T_{H}}{T_{C}} \leq 2\end{cases}
$$

For the phase angle difference $(\phi)$ :

Heater:

$$
f_{h \phi}(\phi)=1.463667 \times 10^{-4} \phi^{2}-1.5516 \times 10^{-2} \phi+0.798
$$

Cooler: $f_{c \phi}(\phi)=7.04762 \times 10^{-5} \phi^{2}-1.504764 \times 10^{-2} \phi+0.201$

Regenerator:

$f_{r \phi}(\phi)=8.0381 \times 10^{-5} \phi^{2}-1.60382 \times 10^{-3} \phi+0.08$

Note: the absolute value of $\phi$ should be used for above equations.

For the dead volumes:

Heater:

$$
f_{h D}= \begin{cases}0.1373 \times D H+0.8864 & ; \mathrm{DH} \leq 2 \\ 0.408 \times \mathrm{DH}+1.08 & ; \mathrm{DH}>2\end{cases}
$$

Cooler: $f_{c D}(D C)= \begin{cases}-0.333 \times D C+1.293 & ; \mathrm{DC} \leq 0.6 \\ -0.111 \times D C+1.163 & ; \mathrm{DC}>0.6\end{cases}$
Regenerator:

$$
f_{r D}(D R)= \begin{cases}1.06 & ; \mathrm{T}_{\mathrm{H}}<556^{\circ} \mathrm{C} \\ 1.06 & ; \mathrm{DRR}<1.17 \\ \frac{1}{30} \times D R+1.072 & ; \mathrm{DR}>1.17 \& \mathrm{~T}_{\mathrm{H}}>556^{\circ} \mathrm{C}\end{cases}
$$

Where:

$$
\begin{aligned}
& D H=\frac{V D H}{V_{C}} \\
& D C=\frac{V D C}{V_{C}} \\
& D R=\frac{V D R}{V_{C}}
\end{aligned}
$$

\section{EVALUATION OF DIFFERENT TYPES OF LOSSES}

In general, the losses due to imperfect components can be divided into three types: those which are in the pressurevolume flow domain, those in the force-velocity domain and those which are in the temperature-entropy domain. Pressure drop in the heat exchangers, which produces power loss, belongs to the first group, while axial conduction and the effect of piston motion are in the third group, and coulumb friction is in second group. In this section different types of losses, which have been realized up to this time are evaluated based on the most recent analytical techniques.

\subsection{Pressure-volume flow domain losses}

Although there are some differences in design configurations of Stirling engine but for analysis all of them can be simplified as shown in Fig. 3. There are five major components: cold space, hot space, cooler, regenerator and heater. In the ideal cycle there are only three components: i.e. there is no heater and cooler in the system. In fact, the hot and cold spaces are surrounded by hot and cold temperature sources.

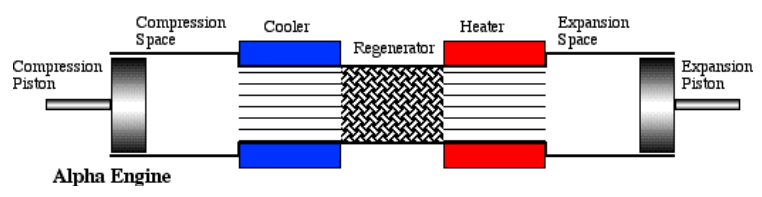

Fig-3: Major components in Stirling engine

Considering the general configurations of a real engine free flow areas in the three heat-exchangers are relatively small which results a significant pressure loss in the system. Power loss due to the pressure drop has been evaluated in a similar fashion in all of the previous analysis.

Pressure drop in three heat exchangers are as follow. 
For Cooler:

$|\Delta P|=\frac{1}{2} \times\left(\frac{L}{d}\right)_{C} \times \frac{R T_{C}}{A F R C^{2}} \times \frac{\dot{f}_{C} \times \dot{m}_{C}}{P_{C}}$

For Heater:

$|\Delta P|=\frac{1}{2} \times\left(\frac{L}{d}\right)_{H} \times \frac{R T_{H}}{A F R H^{2}} \times \frac{\dot{f}_{H} \times \dot{m}_{H}}{P_{H}}$

For Regenerator:

$|\Delta P|=\frac{1}{2} \times\left(\frac{L}{d}\right)_{R} \times \frac{R T_{R}}{A F R R^{2}} \times \frac{\dot{f}_{R} \times \dot{m}_{R}}{P_{R}}$

Where, $\mathrm{P}_{\mathrm{c}}, \mathrm{P}_{\mathrm{H}}$ and $\mathrm{P}_{\mathrm{R}}$ are the mean pressure inside the cooler, heater and regenerator respectively.

Now, supposing the pressure drops have been calculated, how they can be related to the power loss is important yet not quit clear. Proper allocation of the losses and determination of where the entropy generation is occurring both significantly affects the engine performance. Engines which have been presumed to have high efficiencies often in practice do not perform as well. This is most often due to the improper allocation of the losses in the system.

Power loss due to the pressure drop in each heat exchanger, result follows in a closed form solution.

$W_{\text {Loss }}=\frac{f}{8 \pi} \times \frac{L}{R H} \times \frac{\rho}{(A F R)^{2}} \times \frac{V_{C}^{3} \times \omega^{2} \times \sin ^{2} \phi}{\cos ^{2} \alpha} \times F_{c}^{2} \times$

$\left[\sin \phi+\frac{1}{8} \sin (2 \alpha-\phi)+\frac{1}{2} \sin (2 \alpha+\phi)\right]$

Where $\mathrm{f}$ is the friction factor, $\mathrm{A}$ is the flow path area, $\mathrm{L}$ is the length of the heat exchanger and $R H=\frac{A F R \times L}{A H T}$, AHT is the total heat transfer area.

Equation can be re-written for each of the heat exchangers based on their geometries as follows.

\section{For heater:}

$R H=\frac{1}{4} \times D H, \quad \rho=\frac{P_{m}}{R T_{H}}\left(1+\frac{A}{\sqrt{1-A^{2}}}\right)$

$W_{\text {Loss }}=\frac{8}{\pi^{3}} \times f_{H} \times \frac{L_{H}}{D H} \times \frac{V_{C}{ }^{3} \times \omega^{3} \times P_{m} \times\left(1+\frac{A}{\sqrt{1-A^{2}}}\right)}{N_{H}{ }^{2} \times D H^{4} \times R \times T_{H}} \times F_{c h}{ }^{2} \times$

$\frac{\sin ^{2} \phi}{\cos ^{2} \alpha}\left[\sin \phi+\frac{1}{6} \sin (2 \alpha-\phi)+\frac{1}{2} \sin (2 \alpha+\phi)\right]$

Where $\mathrm{DH}$ is the heater tube diameter, $\mathrm{N}_{\mathrm{H}}$ is the number of tubes, $L_{H}$ is the tube length and $f_{H}$ is the friction factor in the heater.

Similarly,

\section{For cooler:}

$$
\begin{aligned}
& W_{\text {Loss }}=\frac{8}{\pi^{3}} \times f_{C} \times \frac{L_{C}}{D C} \times \frac{V_{C}^{3} \times \omega^{3} \times P_{m} \times\left(1-\frac{A}{\sqrt{1-A^{2}}}\right)}{N_{C}{ }^{2} \times D C^{4} \times R \times T_{C}} \times F_{c c}{ }^{2} \times \\
& \frac{\sin ^{2} \phi}{\cos ^{2} \alpha}\left[\sin \phi+\frac{1}{6} \sin (2 \alpha-\phi)+\frac{1}{2} \sin (2 \alpha+\phi)\right]
\end{aligned}
$$

Where DC is the heater tube diameter, $\mathrm{N}_{\mathrm{C}}$ is the number of tubes, $L_{C}$ is the tube length and $f_{C}$ is the friction factor in the heater.

\section{For regenerator:}

$$
\begin{aligned}
& \left(\frac{L}{D}\right)_{R}=\frac{1-\sigma}{\sigma} \times \frac{L_{R}}{d w} \text { (For wire filling) } \\
& R_{H}=\frac{1}{4} \times \frac{1-\sigma}{\sigma} \times d w \\
& \left(\frac{L}{D}\right)_{R}=\frac{3}{2} \times \frac{1-\sigma}{\sigma} \times \frac{L_{R}}{d s} \text { (For sphere fillings) } \\
& R_{H}=\frac{1}{6} \times \frac{1-\sigma}{\sigma} \times d s \\
& A F R R=\frac{V D R}{L_{R}} \\
& W_{L o s s}=\frac{f_{R}}{16 \pi} \times \frac{L_{R}}{R_{H}} \times \frac{P_{m}}{\left(\frac{V D R}{L_{R}}\right)^{2}} \times \frac{V_{C}^{3} \times \omega^{3} \times \sin ^{2} \phi}{\cos ^{2} \alpha \times R \times T_{R}} \times F_{c r}{ }^{2} \times \\
& {\left[\sin \phi+\frac{1}{6} \sin (2 \alpha-\phi)+\frac{1}{2} \sin (2 \alpha+\phi)\right]}
\end{aligned}
$$

\subsection{Temperature-Entropy domain losses}

Events which decrease the effective hot temperature of the hot space and increase the effective cold temperature in the cold space would push the engine farther from the ideal case and considered by investigators up to this time are: temperature drop losses in heater and cooler, regenerator imperfection from heat transfer view point, axial conduction heat transfer loss, piston generate entropy inside the system. Those losses which are in this category and which have been motion or shuttle heat transfer loss, heat leakage from the system, transient heat transfer loss due to non-uniform temperature distribution and heat pumping loss inside the gap between cylinder and piston. In this section derivation of each of these losses is presented.

\subsubsection{Temperature-Drop losses in Heater and Cooler}

A temperature difference is necessary in order to transfer heat between the working fluid and the heat exchanger walls. This means that the temperature of the gas entering the hot and cold spaces and the regenerator will not be the same as heat exchanger wall temperature but a slightly different temperature. 


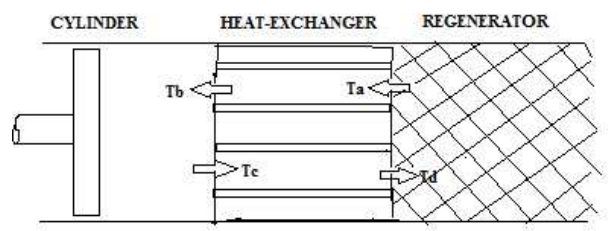

Fig-4: Temperature drop loss in heat-exchanger

The gas enter the heat exchanger from the regenerator with a temperature $\mathrm{T}_{\mathrm{a}}$; at the exit of the heat exchanger the temperature is $\mathrm{T}_{\mathrm{b}}$. when the gas enters the heat exchanger from the cylinder side, its temperature is $\mathrm{T}_{\mathrm{C}}$; at the entrance of the regenerator the temperature is $\mathrm{T}_{\mathrm{d}}$. Further more $\Delta T_{E Q}=T_{c}-T_{b}$. The temperature of the heat exchanger is assumed to remain at $T_{s}$ throughout the cycle. Then from heat exchanger theory.

Effective temperature in Cold space:

$\dot{T}_{C}=T_{C} \times \frac{W_{C} \times(k-1)}{m_{C} \times R \times T_{C} \times k} \times \frac{1}{\exp (2 \times N T U c)-1}$

Effective temperature in hot space:

$\dot{T}_{H}=T_{H} \times \frac{W_{H} \times(k-1)}{m_{H} \times R \times T_{H} \times k} \times \frac{1}{\exp (2 \times N T U h)-1}$

These effective temperatures which are different from the source temperatures $T_{C} \& T_{H}$ would represent temperature drop in heater and temperature increase in cooler. The consequent entropy generation in these heat exchangers results in a power loss which is taken out in the compressor. This means that the compressor work would be increased by a factor $\left(\frac{\dot{T}_{C}}{T_{C}}\right) \times\left(\frac{T_{H}}{\dot{T}_{H}}\right)$.

This means:

Power loss to the temperature drop in heater $W_{\text {lossh }}=\frac{W_{h} \times W_{c} \times(k-1)}{m_{h} \times R \times T_{H} \times k} \times \frac{1}{\exp (2 \times N T U h)-1}$

Power loss to the temperature drop in cooler $W_{\text {lossc } T}=\frac{W_{c} \times W_{c} \times(k-1)}{m_{c} \times R \times T_{C} \times k} \times \frac{1}{\exp (2 \times N T U c)-1}$

\subsubsection{Imperfect heat transfer in regenerator}

In the ideal (perfect) regenerator when gas flows through the regenerator coming from the warm-end of the engine at hot temperature $T_{H}$, it increases heat such that by the time that the flow leaves the regenerator it reaches the cold temperature $T_{C}$ and the regenerator gains all of the heat. Then similarly, when gas flows back from the cold-end at $\mathrm{T}_{\mathrm{C}}$ temperature and back through the regenerator it should be reheated by the regenerator such that when the flow leaves the regenerators it should have temperature $\mathrm{T}_{\mathrm{H}}$. This happens only in ideal case, i.e. having instantaneous heat transfer with no thermal resistances. Therefore, we can define regenerator effectiveness as the ratio of the actual heat which is transferred to the gas by the regenerator to that transferred ideally.

$$
E=\frac{Q_{R}}{m \times C_{p}\left(T_{H}-T_{C}\right)}
$$

If the effectiveness can be calculated then $\mathrm{Q}_{\mathrm{R}}$ and consequently $m \times C_{p}\left(T_{H}-T_{C}\right)-Q_{R}$ which the heat loss due to the imperfect regenerator can be evaluated. Qvale and smith [3] have derived on approximate closed form solution for the enthalpy flow through the regenerator. This solution was derived sinusoidal pressure and flow variations. Rios [4] derived a more general solution for this enthalpy flow calculations but it requires computer simulations.

$$
\begin{aligned}
& Q_{R}=f_{t} \times \dot{m}_{R} \times C_{v}\left(T_{H}-T_{C}\right) \times \frac{2}{N T U_{r}+2} \\
& \text { Where }{ }_{N T U_{r}}=\frac{H \times A H}{\dot{m}_{R} \times C_{v}}
\end{aligned}
$$

\subsubsection{Axial conduction heat loss}

All materials conduct heat to a greater or lesser extent. Metals are generally good conductors. Since there is a temperature gradient between the two ends of the Stirling engine, then one might expect a significant amount of heat conduction from the warm side of the system (heater) to the cold side (cooler). The intermediate which conduct this heat are regenerator internal solid materials are also the gas inside the void volume of the regenerator. Usually the regenerator of a Stirling engine is made of many layers fine screen stack since the controlling resistance is now the contact between adjacent wires. Since the wires are somewhat like uniformly sized cylinders then the formula which is given by Gorring [5] can be used for thermal conductivity of the combination of gas and material of the combination of gas and material of the regenerator.

$$
k_{m g}=k_{g} \times \frac{\frac{1+\frac{k_{m}}{k_{g}}}{1-\frac{k_{m}}{k_{g}}}-1+\sigma}{\frac{1+\frac{k_{m}}{k_{g}}}{1-\frac{k_{m}}{k_{g}}}+1-\sigma}
$$

Where $\sigma$ the regenerator porosity, $\mathrm{k}_{\mathrm{g}}$ is the gas conductivity and $\mathrm{k}_{\mathrm{m}}$ is the metal conductivity. Therefore the conducted heat would be 
$Q_{C}=k_{m g} \times A R \times \frac{T_{H}-T_{C}}{L_{R}}$

Where $L_{R}$ is the regenerator length and AR is the crosssectional area of the regenerator.

\subsubsection{Shuttle heat transfer loss}

Shuttle loss is one of the important thermal effects found in Stirling engine. This effect comes from the reciprocating action of the displacer (piston) from the hot end to the cold end.

Top Dead Centre

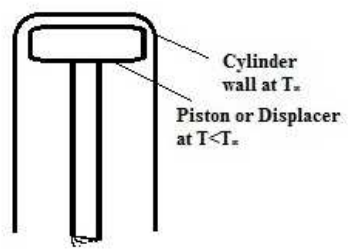

Bottom Dead Centre

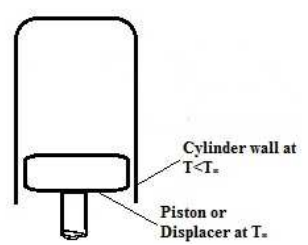

Fig.5 Shuttle heat transfer loss

When the displacer (piston) moves toward the top dead centre position its temperature is less than $\mathrm{T}_{\mathrm{H}}$ and it is entering to a region with $T_{H}$ temperature. Therefore there is a heat conduction from the cylinder walls to the displace (piston) is at bottom dead centre. This conduction occurs; through each reciprocation. Rios [4] has presented a nice derivation for this heat conduction.

$Q=\frac{\pi}{8} k_{g} \times s \times\left(T_{H}-T_{C}\right) \times\left(\frac{B}{l}\right) \times\left(\frac{S}{L}\right) \times B E T$

$B E T=\frac{2 C^{2}-C}{2 C^{2}-1}$

$C=\frac{k_{p}}{k_{g}} \times l \times \sqrt{\frac{\omega}{2 \times \alpha_{p}}}$

Where B and s are the bore and stroke of the hot cylinder; $\mathrm{k}_{\mathrm{p}}$ and $\alpha_{\mathrm{p}}$ are thermal conductivity and thermal diffusivity of the displacer (piston); 1 is the gap between piston and cylinder; $\mathrm{L}$ is the piston length and $\omega$ is the frequency of the engine.

\subsubsection{Heat leakage}

Since the warm-end of the system operates at a temperature greater than the ambient temperature, then heat conduction and radiation effects are present. Depending on the hot temperature if $\mathrm{T}_{\mathrm{H}}$ is high enough i.e. of the order of $810 \mathrm{~K}$ then radiation heat transfer would be significant otherwise conduction would be the primary mechanism for significant heat leakage from the system. Because the present work deals with the temperature ratios less than 2.0, then only conduction heat transfer is assumed.

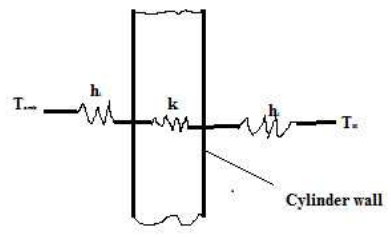

Fig.6 Heat leakage in cylinder wal 1

$$
Q_{L}=\frac{2 \pi\left(T_{H}-T_{a m b}\right)}{\frac{1}{L \times R \times h_{i}}+\ln \frac{\left(\frac{R+t}{R}\right)}{L \times k_{c}}+\frac{1}{L \times(R+t) \times h_{o}}}
$$

For forced convection inside the cylinder we take $\frac{h_{i} \times D}{k_{g}}=6$ $\Rightarrow h_{i}=\frac{3 \times k_{g}}{R}$

For free convection outside, especially because the outside fluid is air, we assume $h_{0}=0.019874 \frac{\mathrm{kJ}}{\mathrm{m}^{2} \times s \times K}$. Also for length $\mathrm{L}$ one half of the stroke is chosen. This is due to the fact that gas has almost a sinusoidal motion the amplitude of that which is half of the stroke would be a suitable characteristic of length. Therefore, by substituting all of these we get:

$$
Q_{L}=\frac{\pi\left(T_{H}-T_{a m b}\right)}{\frac{1}{3 s \times k_{g}}+\ln \frac{\left(\frac{R+t}{R}\right)}{s \times k}+\frac{1}{3.5 s \times(R+t)}}
$$

Where $\mathrm{k}_{\mathrm{g}}$ and $\mathrm{k}_{\mathrm{c}}$ are thermal conductivities of the gas inside the cylinder and the wall material respectively, $\mathrm{s}$ is the stroke and $\mathrm{R}$ is one half of the bore.

If the radiation heat transfer is significant then a similar analysis with radiation heat transfer equation can be used. Although the temperature inside the cylinder has a sinusoidal variation with a mean value, i.e. it is a combination of a constant and an alternatory temperature but because of low amplitude of the alternatory part, less than $10 \%$ of the constant 
value, the mean (or constant) value can be used in this equation for heat leakage calculations.

\subsubsection{Transient heat transfer loss in the cylinders}

One of the important characteristics of an ideal Stirling engine is the assumed uniform temperature distribution of the gas inside the cylinders. However, in a real engine the temperature is not uniformly distributed at any time inside the cylinders. The resultant temperature gradient between the layers of the gas in the centre and near the wall of the cylinder produces a heat flow from the wall to the centre and vice versa, which Smith and Co-writer have called the instantaneous or transient heat transfer.

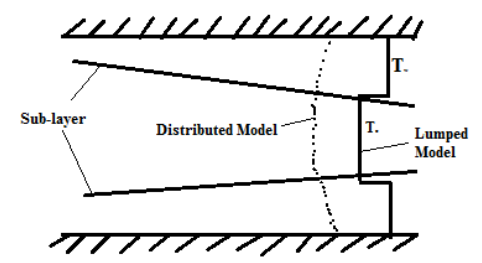

Fig-7: Transient Heat transfer Lump model

The result becomes:

$Q=.052 \times(\mathrm{Re})^{.8}(p r)^{.4}\left(\frac{k-1}{k}\right) \times \frac{P_{l}}{P_{m}} \times \cos \phi_{p} \times k_{g} \times T_{m} X_{m}$

Where $X_{m}$ is the amplitude of the piston displacement which equals to one-half of the stroke.

\subsubsection{Heat pumping loss inside the piston and cylinder gap}

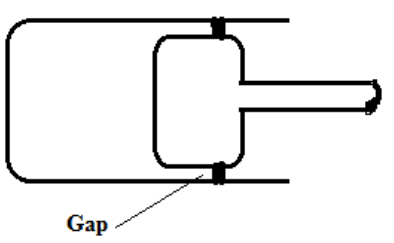

Fig-8: pumping heat loss inside the piston and cylinder gap

There is a radial gap between the inner diameter (ID) of the engine cylinder and the outer diameter (OD) of the piston (Displacer). This gap is ring sealed at the cold end. As the engine is pressurized and depressurized gap flows into and out of this gap. Since the closed end of the gap is cold, extra heat must be added to the gap as it comes back from this gap. Leo [5] has derived the following semi-empirical equation for the amount of heat loss by this mechanism

$Q_{o s s}=\frac{2 \omega}{3 \pi} \times\left[\frac{\omega B C_{p}}{2 k_{g}}\right]^{6} \times\left[\frac{\left(P_{\max }-P_{\min }\right) \times l}{R \times T_{R}}\right]^{1.6} \times C_{p}\left(T_{H}-T_{C}\right) \times L \times L_{p}$
Where $\mathrm{B}$ is the bore, 1 is the gap side, $\mathrm{L}_{\mathrm{p}}$ is the length of the displacer (piston) and $\omega$ is the frequency.

\subsection{Force Displacement domain loss}

The only utilized loss in this category is mechanical frictional loss. in the literature of Stirling engine there is no equation for correlation or even any data for this type of loss. Martini [5] has arbitrarily used $20 \%$ of the basic output power as mechanical fraction. For the present analysis the data for internal combustion engines has been used to estimate this loss. Edward [10] derived correlation for mechanical friction loss for the piston in term of the friction mean effective pressure $\left(f_{\text {mep }}\right)$. Since it approximates a linear relationship between $f_{\text {mep }}$ and engine speed, then the following correlations has been used.

$f_{\text {mep }}=6.8948 \times 10^{-2} \times(.002 N+1)$

Where $\mathrm{N}$ (RPM) and $\mathrm{f}_{\text {mep }}$ (bar).

Then the power loss due to this friction effect, based on the mean effective pressure definitions, is:

$p=f_{\text {mep }} \times \frac{V \times N \times n}{531}$

Where $\mathrm{n}$ is number of cylinders and $\mathrm{V}$ is the displacement volume

$p_{\text {loss }}=2 \times 14.6706 \times(.002 N+1) \times\left(V_{H}+V_{C}\right)$

$\mathrm{N}$ in RPM, $\mathrm{V}_{\mathrm{H}}, \mathrm{V}_{\mathrm{C}}$ in $\mathrm{cc}$ and $\mathrm{P}_{\text {loss }}$ in Watts.

\section{AVAILABLE DATA AND COMPARISON WITH COMPUTATIONAL MODELS}

Using all this analysis for behavior prediction of Stirling engine one mathematical model created in C-language programming that called Computational model. The input data for this model are: temperature of hot and cold sources, mean pressure inside the system, working fluid, speed of the engine, cold and hot space volumes, and dead volumes, phase angle difference between the hot and cold spaces, volume displacements and the heat exchangers geometries. This Computational model can be used for any operational temperature ratio and mean pressure. Since the low temperature ratio and mean pressure. Since the low temperature ratio results are to be compared with the available data in the following chapters, then in this section we use the certain high temperature measured data on Stirling engines to compare with the complete model predictions. In this way, we might better test the accuracy of this model over a wide operating range for temperature and temperature ratio. 
Many different types of Stirling engines have been designed and built. However, only a few of them have been published specifications wherein sufficient details have been given in the literature. For the cooperation purposes of this section the following engines are considered and discussed.

\section{(A) Allison engine}

The investigation conducted by the Allison division of General Motors Corporations [10] has been the only test reported in which all conditions were described in sufficient detail to give a reliable verifications of the overall model. The development of this engine was part of a space-power program and several engine designs were built and tested as part of the project. The recorded performances data of two of the designs are compared to the results of the Computational model.

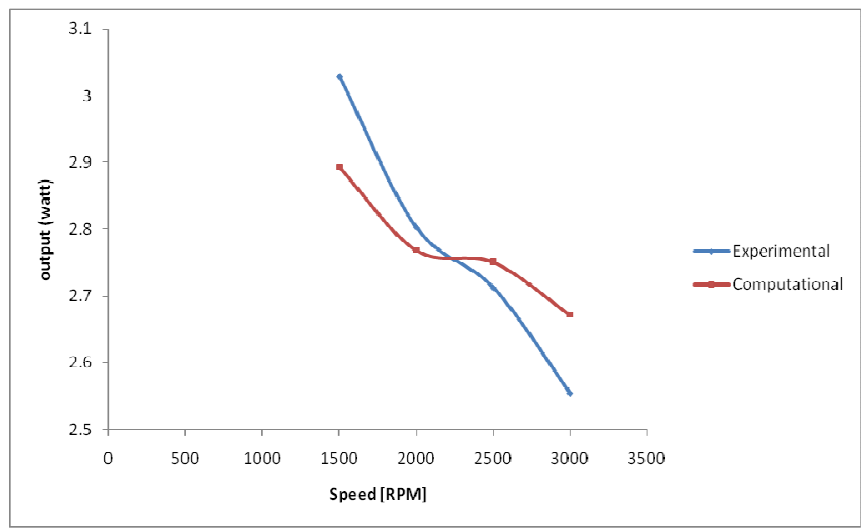

Fig-9: Comparison of Allison engine output with Computational model $(\Phi=118)$

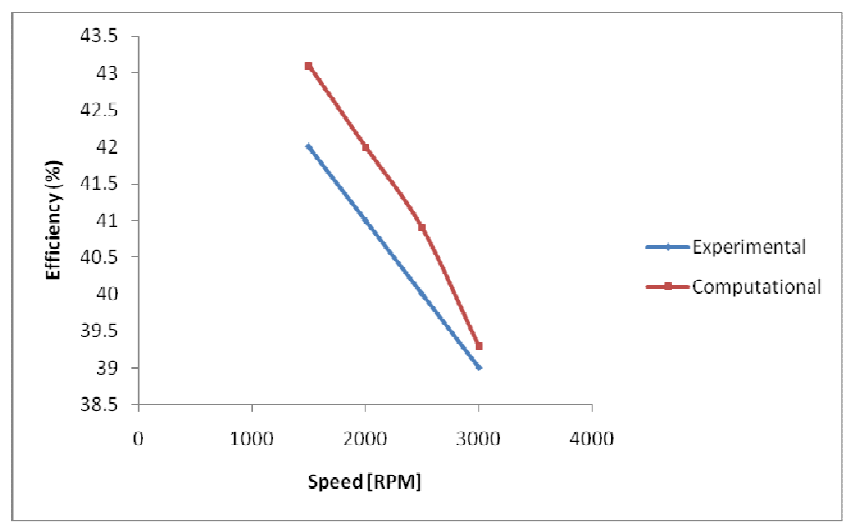

Fig-10: Comparision of Allison engine efficiency with Computational model (phy=118)

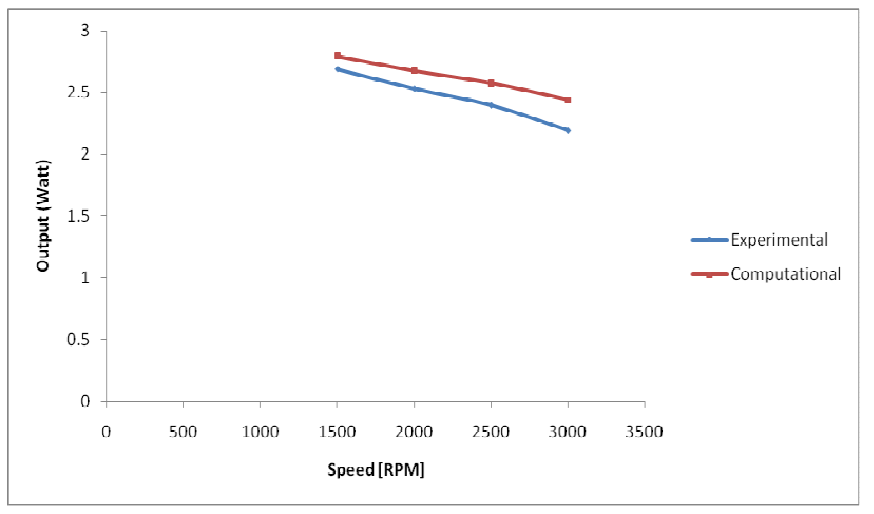

Fig-11: Comparison of Allison engine output with Computational model $(\Phi=112)$

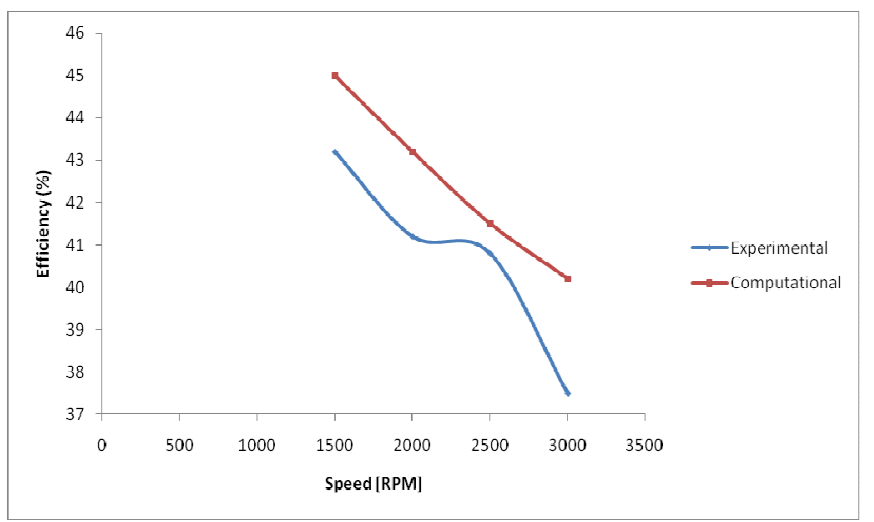

Fig-12: Comparison of Allison engine efficiency with Computational model $(\Phi=112)$

\section{(B) GPU-3 engine:}

General Motors Research conducted a program for the U.S. Army to produce a silent electric power source in 1960s. This Ground power unit (GPU) development went through three different models. Two of the last model GPU-3 were preserved and are now being used by NASA Lewis Research Centre to obtain reliable measurements of a more or less modern type of Stirling engine.

Lewis Research Centre (LeRC) has used a third order analysis and presented a series of test points with computed performances. Therefore the results of LeRC are compared with the complete model results in Fig. 13 and 14. This comparison shows that LeRC model is more optimistic than the present Computational model from the output power view point. On the other hand their heat input is more than that what the Computational model is predicting. This is due to the fact that the Computational model has been run with incomplete specifications of the engine. Such as dead volumes inside the 
system and that is the reason for having higher efficiencies from the Computational model than LeRC results. Finally the LeRC performances are assuming the mechanical dry friction losses to be $20 \%$ of the basic output power, which has no reason to be a good approximation.

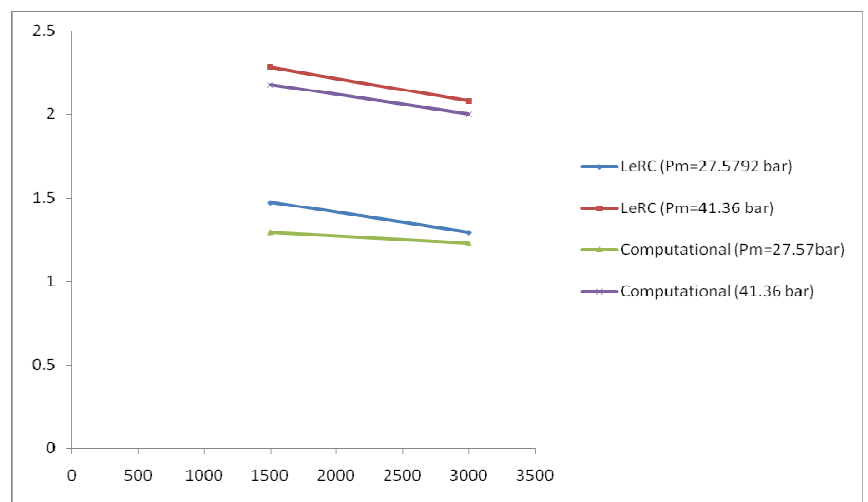

Fig-13: Comparison of GPU-3 engine output with Computational model $\left(\mathrm{H}_{2}\right)$

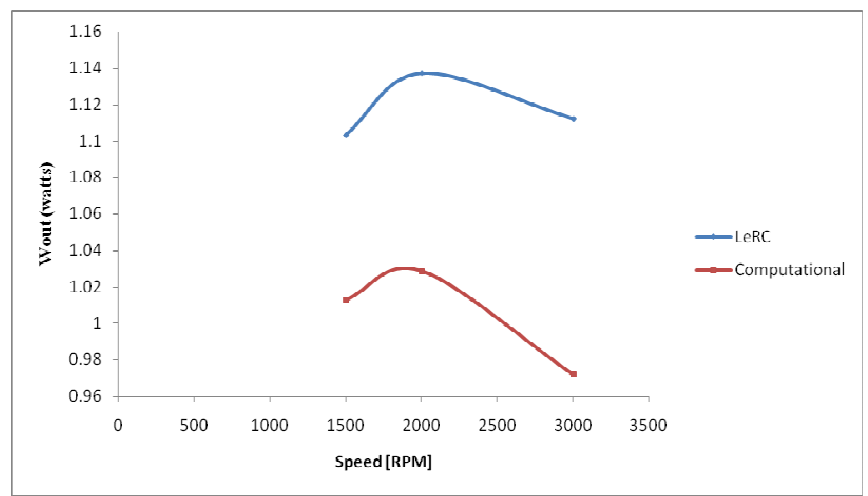

Fig-14: Comparison of GPU-3 engine output with Computational model $\left(\mathrm{He}_{2}\right)$

\section{CONCLUSION}

The Computational model has been successfully applied to the analysis and prediction of the performance of practical Stirling. This requires no "real times" calculations. Merely by giving the operating conditions and engine geometry the model yields the performance of the engine with detailed values of the different losses.

\section{REFERENCES}

[1] G. Walker, "Stirling engines", Oxford Science Publications 1980.
[2] R. K. Rajput, "Heat and mass transfer", S. Chand, New Delhi, 2007.

[3] Einar B., Qvale and J. L. Smith Jr, "An Approximate solution for the Thermal performance of the Stirling engine Regenerator", ASME paper, 68-WA-Enter-1,1968.

[4] Perdro A. Rios, "An Analytical and experimental investigation of the Stirling cycle", D.Sc., Thesis, M.I.T. 1969.

[5] W. R. Martin, M. L. Collie, "Stirling engine design and feasibility for automotive use", Energy technology, Review no.47. 1979.

[6] W.J.D. Annand, "Heat transfer in the Cylinders of Reciprocating internal engines", Proceedings of institutions of mechanical engineers, volume 177, No. 36, 1963.

[7] R. P. Adair, E. Qvale"Instantneous heat transfer to the cylinder wall in reciprocating compressors ", proceedings of 1972 purdue compressor tech. conference.

[8] Kanppil Lee, Joseph L., Smith Jr., Henry B. Faulkner, "performance loss due to transient heat transfer in the cylinder of Stirling engines", $15^{\text {th }}$ IECEC, paper 799251, August 1979.

[9] C. Fayette Taylor, Edward S. Taylor, "The internal combustion engine”, International Text Book Co., 1966.

[10] Einar B., Qvale, “An analytical model of Stirling type engines”, Ph.D. Thesis, M.I.T. January 1967.

\section{BIOGRAPHIES}

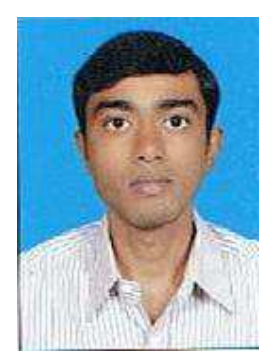

Name: Mr. Rakesh K. Bumataria

Contact No: +91-9924402808

E-mail:

bumatariarakesh@yahoo.co.in

Specialization: Thermal

Analysis, CFD, Fluid Flow

Analysis

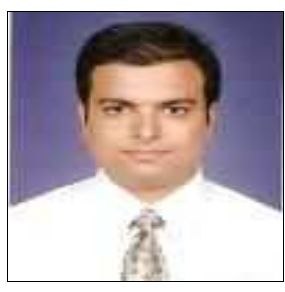

Name: Mr.Nikul K. Patel

Contact No: +91-9909976040

E-mail: nikulatmsu@gmail.com

Specialization: I C Engine, Automobile, Bio-diesel, CFD Analysis 